\title{
Evaluation and Treatment of Inherited Pheochromocytoma in Pregnancy
}

Alison E Thompson, Benjamin C James, Brian David Saunders

\begin{abstract}
Pheochromocytomas represent a rare, but potentially devastating, cause of hypertension among pregnant patients. Inherited predisposition to these tumors requires vigilant screening, especially in women of child-bearing age. We present an illustrative case of a multiple endocrine neoplasia type $2 \mathrm{~A}$ patient who was successfully surgically treated for a pheochromocytoma during pregnancy. A review of the existing, English-language literature and screening guidelines was undertaken. Pheochromocytomas presenting during pregnancy, can be safely and effectively treated with laparoscopic surgery, allowing the maintenance of a normal pregnancy. Screening of patients with inherited endocrinopathies that predispose to adrenal medullary tumors must be adapted to maximize the opportunity to detect a pheochromocytoma in the pregravid state. More frequent testing and a lower threshold for biochemical or radiologic investigation are suggested for women in their reproductive years.
\end{abstract}

Keywords: Pheochromocytoma, MEN-2A, Pregnancy screening.

Abbreviation: MEN-multiple endocrine neoplasia; RET-rearranged during transfection; MRI-magnetic resonance imaging.

How to cite this article: Thompson AE, J ames BC, Saunders $B D$. Evaluation and Treatment of Inherited Pheochromocytoma in Pregnancy. World J Endoc Surg 2013;5(3):82-84.

\section{Source of support $\mathrm{Nil}$ \\ Conflict of interest None}

\section{INTRODUCTION}

Though pheochromocytomas in pregnant women are rare, with an incidence of approximately 1 in 50,000 pregnancies, these tumors can lead to significant morbidity and potential mortality for both mother and fetus. ${ }^{1}$ Because pheochromocytomas secrete catecholamines such as epinephrine and norepinephrine, the mother may experience heart dysrhythmias, hypertensive crises or seizures. ${ }^{2}$ Furthermore, as excess catecholamines circulate in the maternal vasculature, the uteroplacental vascular bed is at risk of excess vasoconstriction, which can lead to intrauterine hypoxia or placental abruption. ${ }^{3}$ If left untreated, mortality rates for both mother and fetus have been reported as high as $58 \% .{ }^{4} U$ nfortunately, the diagnosis of pheochromocytoma may be missed in pregnant patients due to the tumor's ability to mimic other forms of gestational hypertension. Therefore, it is important to keep pheochromocytoma on the differential diagnosis for a gravid woman who develops palpitations, headaches, or spikes of high blood pressure. This is especially true in a patient with a known or suspected inherited endocrine tumor predisposition syndrome such as multiple endocrine neoplasia (M EN) type $2 .^{5}$

The current screening guidelines for hereditary pheochromocytomas suggest annual biochemical screening with plasma metanephrine and normetanephrine levels. ${ }^{2}$ A known RET proto-oncogene mutation, such as occurs in $M E N-2 A$ and $M E N-2 B$, should prompt screening beginning at age 5 . When metanephrine levels are greater than $2 x$ the upper limit of normal, imaging is indicated to localize the tumor. ${ }^{6}$ The current imaging method of choice for pregnant women is a noncontrast M RI of the abdomen, specifically focusing on the adrenal glands. ${ }^{4}$ Once imaging confirms the diagnosis, patients are often referred for surgical intervention; in pregnant women, laparoscopic adrenalectomy can be performed during the second trimester. ${ }^{1}$ However, surgery during pregnancy is not without risks. The following case demonstrates ways in which the current screening guidelines could be improved with regards to women of reproductive age with inherited endocrine tumor syndromes which greatly increase the likelihood of developing a pheochromocytoma.

\section{CASE REPORT}

We report a 25-year-old female with M EN-2A secondary to a Ret codon 634 mutation who was undergoing routine surveillance by her primary physician for adrenal medullary hyperfunction. Laboratory evaluation showed a rise in urinary catecholamines, but further endocrinology and endocrine surgery consultation was not sought for several months until levels were greater than $2 x$ the upper limit of normal upon repeat biochemical interrogation. At this point, however, the patient was also 18 weeks pregnant. Confirmatory plasma testing reveal ed el evated fractionated metanephrines $(136 \mathrm{pg} / \mathrm{ml},<57 \mathrm{pg} / \mathrm{ml})$ and total metanephrines $(277 \mathrm{pg} / \mathrm{ml},<205 \mathrm{pg} / \mathrm{ml})$, and a noncontrast abdominal MRI revealed a $2.2 \mathrm{~cm}$ right adrenal mass suspicious for pheochromocytoma (Fig. 1). The patient was asymptomatic, but had relative systolic hypertension (SB Ps in the 120s; baseline of 90s). The patient was prepared for operative resection with 10 days of oral phenoxybenzamine 
(10 mg once daily) and oral metoprolol (25 mg twice daily). $M$ aternal fetal medicine consultation was obtained perioperatively. The patient was admitted to the hospital 3 days preoperatively for intravenous hydration given the therapeutic alpha blockade. The patient underwent an uncomplicated, transabdominal laparoscopic right adrenalectomy at 22 weeks of gestation. There were no intraoperative hemodynamic perturbations. Total operative time was 64 minutes, and the estimated blood loss was less than $50 \mathrm{cc}$. Her postoperative course was uneventful, and she was discharged on postoperative day number one. Pathology confirmed a pheochromocytoma. Repeat biochemical testing at 31 and 36 weeks of gestation showed no further evidence of catechol excess. The patient went on to deliver a healthy baby girl via a normal spontaneous vaginal delivery at 41 weeks gestation.

\section{DISCUSSION}

Pheochromocytomas and especially those associated with MEN-2, in pregnancy are rare, yet can have significant morbidity and mortality for the mother and the fetus. Though this case illustrates the successful multidisciplinary medical and surgical treatment of pheochromocytoma in a pregnant woman, surgery during pregnancy does incur greater risks and more complex perioperative care to ensure the safety of mother and fetus. As with any surgical procedure, there are risks for complications such as bleeding, infection, and poor wound healing; blood pressure control is additionally of vital importance as tumor mani pulation during extirpation can potentially lead to hemodynamic lability. ${ }^{7}$ Excessive or prolonged elevations of catecholamines can be detrimental to both mother and fetus, and caution must be taken during tumor extirpation. Therefore, in at-risk populations where

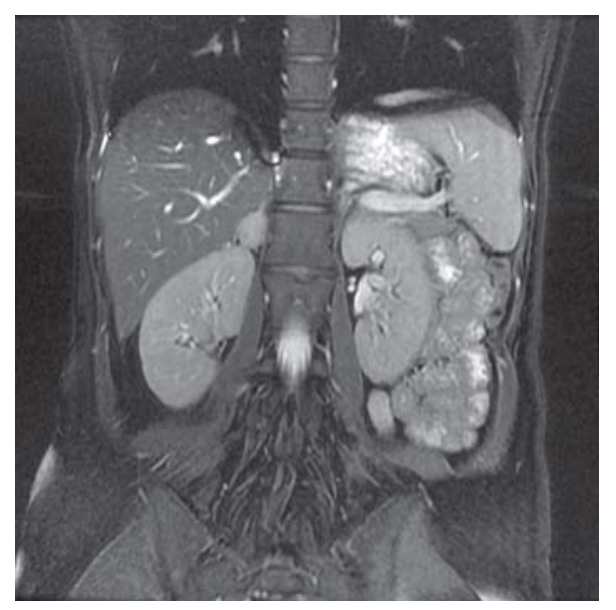

Fig. 1: Coronal section of a non-contrast abdominal MRI revealing a $2.2 \mathrm{~cm}$ right suprarenal mass consistent with pheochromocytoma the likelihood of developing a pheochromocytoma is greatly increased, such as those with multiple endocrine neoplasia type 2 , detection of such tumors would ideally occur in a nongravid patient.

At the current time, there have only been 8 reported cases of pregnant women with MEN-2A who have undergone adrenalectomy for pheochromocytomas during pregnancy. Both due to the rarity of such an encounter, as well as the increased complexity of the procedure, adrenalectomy may be daunting to even the most experienced surgeon. W e assert that laparoscopic adrenalectomy for pheochromocytoma performed during pregnancy is safe and effective.

Current screening guidelines for MEN-2 and other inherited pheochromocytoma syndromes do not specifically address female patients of reproductive age. We suggest that screening guidelines should be adjusted for inherited pheochromocytomas in women of reproductive age.

Though a diagnosis of pheochromocytoma with catecholamine levels less than 2 to 3 times the upper limit of normal is unlikely for most patients, this rule may not apply to the population of patients with known inherited endocrine disorders. ${ }^{8}$ In the case presented herein, the patient had mild increases in circulating fractionated metanephrines documented prior to those levels that spurred further workup. Though waiting and reassessing biochemical parameters until such time as they are above a pre-set threshold before expanding the patient's evaluation is the current recommendation, we suggest that there needs to be a lower threshold for further testing in women of reproductive age that are at risk for familial catecholamine-secreting tumors. Further, women of child-bearing age would benefit from semi-annual screening rather than just annual assessment. M ortality rates are high for both mother and fetus with untreated pheochromocytomas. By having heightened suspicion in this specific population, we hope to better avoid treating these tumors during pregnancy. Rather than waiting for biochemical results to reach the designated cut-off, further workup for any elevations in plasma or urine catecholamines may save a patient from undergoing surgery during pregnancy.

\section{CONCLUSION}

Pheochromocytoma poses a significant threat to two lives when occurring in pregnant women. Though the incidence of such cases is rare, women with inherited endocrine syndromes are at greater relative risk of developing catecholamine-secreting tumors, especially during the childbearing years. Heightened vigilance to and follow-up for 
any biochemical abnormality is required when screening women of child-bearing age for familial catecholaminesecreting tumors.

\section{REFERENCES}

1. Gomez AZ, Polo MAA, Martin MA, et al. Management of pheochromocytoma during pregnancy: laparoscopic adrenalectomy. The A merican Surgeon 2012;78:E156-E158.

2. Machens $A$, Lorenz K, Dralle H. Peak incidence of pheochromocytoma and primary hyperparathyroidism in multiple endocrine neoplasia 2: Need for age-adjusted biochemical screening. Journal of Clinical Endocrinology M etabolism 2013 98(2):E336-E345.

3. L enders J W M . Endocrine disorders in pregnancy: pheochromocytoma and pregnancy: a deceptive connection. European J ournal Endrocrinology 2012;166:143-150.

4. Oliva R, Angelos P, Kaplan E, et al. Pheochromocytoma in pregnancy: a case series and review. Hypertension 2010;55: 600-606.

5. Biggar MA, Lennard TW J. Systematic review of phaeochromocytoma in pregnancy. B ritish Journal of Surgery 2013 100(2):182-190.
6. Lenders JW, Eisenhofer $G, M$ annelli $M$, et al. Phaeochromocytoma. Lancet 2005;366(9486):665-675.

7. Gaujoux S, B onnetS, Leconte M , et al. Risk factors for conversion and complications after unilateral laparoscopic adrenalectomy. B ritish J ournal of Surgery 2011;98(10):1392-1399.

8. Sarathi V, Lila AR, B andgar TR, et al. Pheochromocytoma and pregnancy: a rare but dangerous combination. Endocrine Practice 2010;16(2):300-309.

\section{ABOUT THE AUTHORS}

\section{Alison E Thompson}

Medical Student, Department of Surgery, Penn State College of M edicine, Pennsylvania, U nited States

\section{Benjamin C James}

Endocrine Surgery Fellow, Department of Surgery, University of Chicago M edical Center, Pennsylvania, U nited States

\section{Brian David Saunders (Corresponding Author)}

A ssistant Professor, Department of Surgery, Penn State College of M edicine, Pennsylvania, U nited States, Phone: 7175315272 e-mail: bsaunders@hmc.psu.edu 